\title{
Holographic read-only memory
}

\author{
G. Z H O U, D. P S A L T S A N F . M O K \\ Holoplex Inc.600 S. Lake Ave., Ste.102, Pasadena, CA 91106,USA (E-mail: gan@holoplex.com)
}

\begin{abstract}
We describe the three-dimensional holographic optical disc system for application as read-only memory. Several systems design issues are discussed including imaging optics, storage density, readout rate, and the alignment servo system. We demonstrate $40 \mathrm{bits} / \mu^{2}$ surface density and pixel-matched holographic data readout from a $12 \mathrm{~cm}$ disc spinning at 600 RPM.
\end{abstract}

Key words: holographic storage, read-only memory, readout, storage density

\section{Introduction}

The holographic read-only memory is a high capacity, disc-based data storage device that can provide the performance needed for next generation mass data storage ( $\mathrm{Li}$ and Psaltis 1994; Psaltis and Mok 1995; Psaltis and $\mathrm{Pu}$ 1995; Zhou et al. 1998). With a projected capacity approaching 1 terabit on a single $12 \mathrm{~cm}$ platter, the holographic disc has the potential to replace CD-ROM jukeboxes as the storage hardware for large databases. The high readout rate of holographic disc makes it especially suitable for applications in a network environment where a server must generate multiple, high bandwidth data streams to client computers. Because of the holographic nature of the stored data, the holographic disc can also offer unique advantages in various data warehousing and data mining applications.

Figure 1 is the conceptual diagram of data readout from a holographic disc. The system stores digital holographic images on a flat, disk-shaped medium. Stored images are organized in different tracks. The disc can spin continuously and it can also move across tracks to allow the laser pickup to access the entire surface area of the medium. For each storage location, many angle-multiplexed holograms are recorded and overlapped.

The complete holographic disc-based ROM system consists of three separate modules. The first is the recording module. This is where the holograms are recorded onto a fresh disc. This module includes both the reference and signal beam paths and it contains components such as the SLM and the scanners. The recording module is similar to the CD-ROM mastering device, and as such it could be large and bulky if necessary. The second is the readout module which was shown in Fig. 1. This is where the holographic images are retrieved from the disc and converted to electrical signals by an appropriate detector array. This module contains light modulators, scanners, 


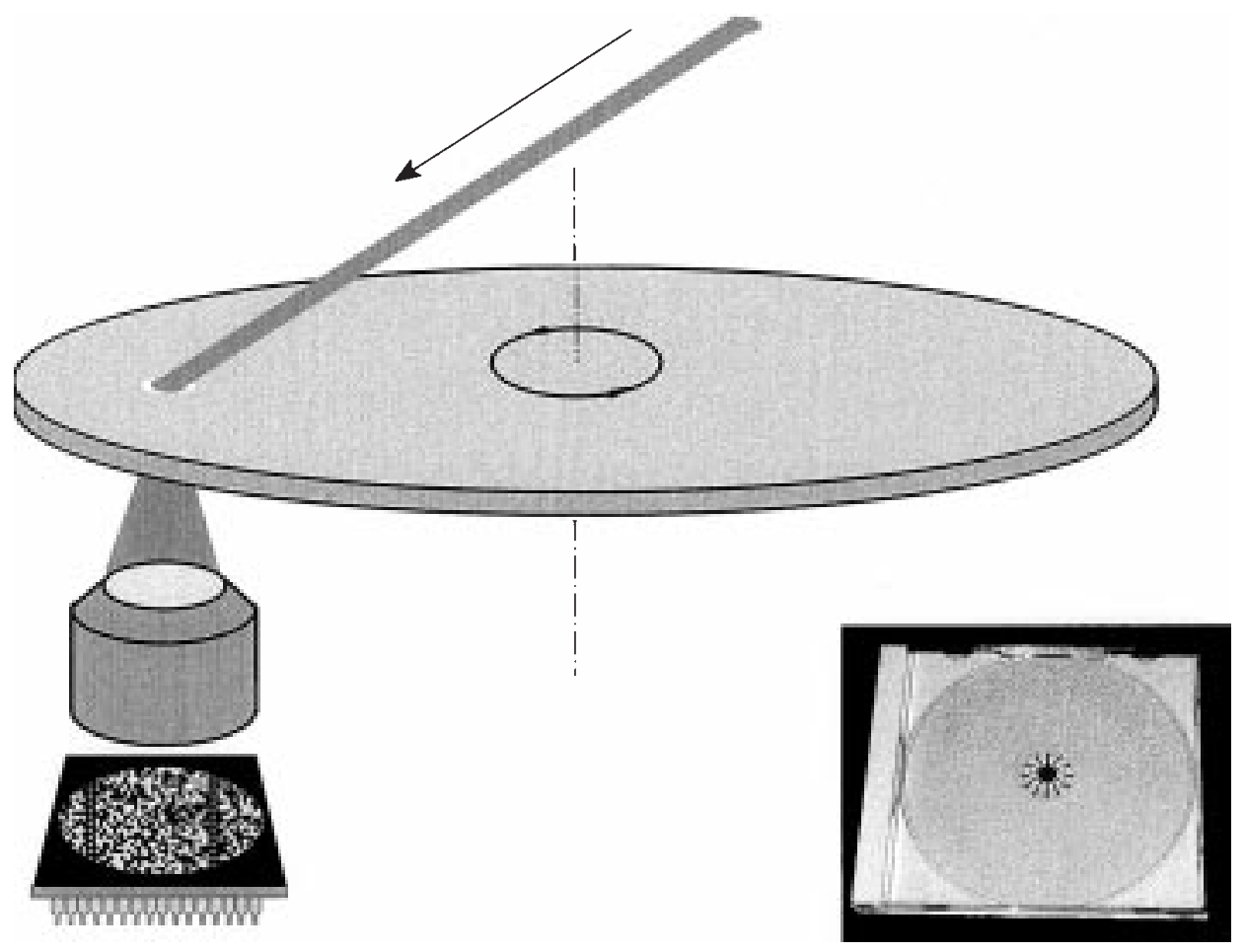

Fig. 1. Illustration of the 3D holographic disc system.

and other electronics. The readout module should be compact and portable. The third module is a device to replicate the recorded master hologram and efficiently produce copy discs in volume. In this paper we will focus the discussion the technical aspects of the recording module and the readout module.

\section{The recording setup}

Figure 2 is the schematic drawing of the recording module for holographic disc system. The setup consists of a diode pumped green laser source at $532 \mathrm{~nm}$. The numerous half-wave plates and polarizers surrounding the polarized beam splitter allows us to transfer power between the two arms. In the signal arm, an SLM is illuminated by a plane wave, and the disc to be recorded is located at the Fourier plane of the SLM. A detector array is placed at the image plane of the SLM. In the reference arm, a beam deflector and a 4- $f$ imaging system are used to scan the angle of the reference beam to implement angular multiplexing. During recording, the deflector scans the 


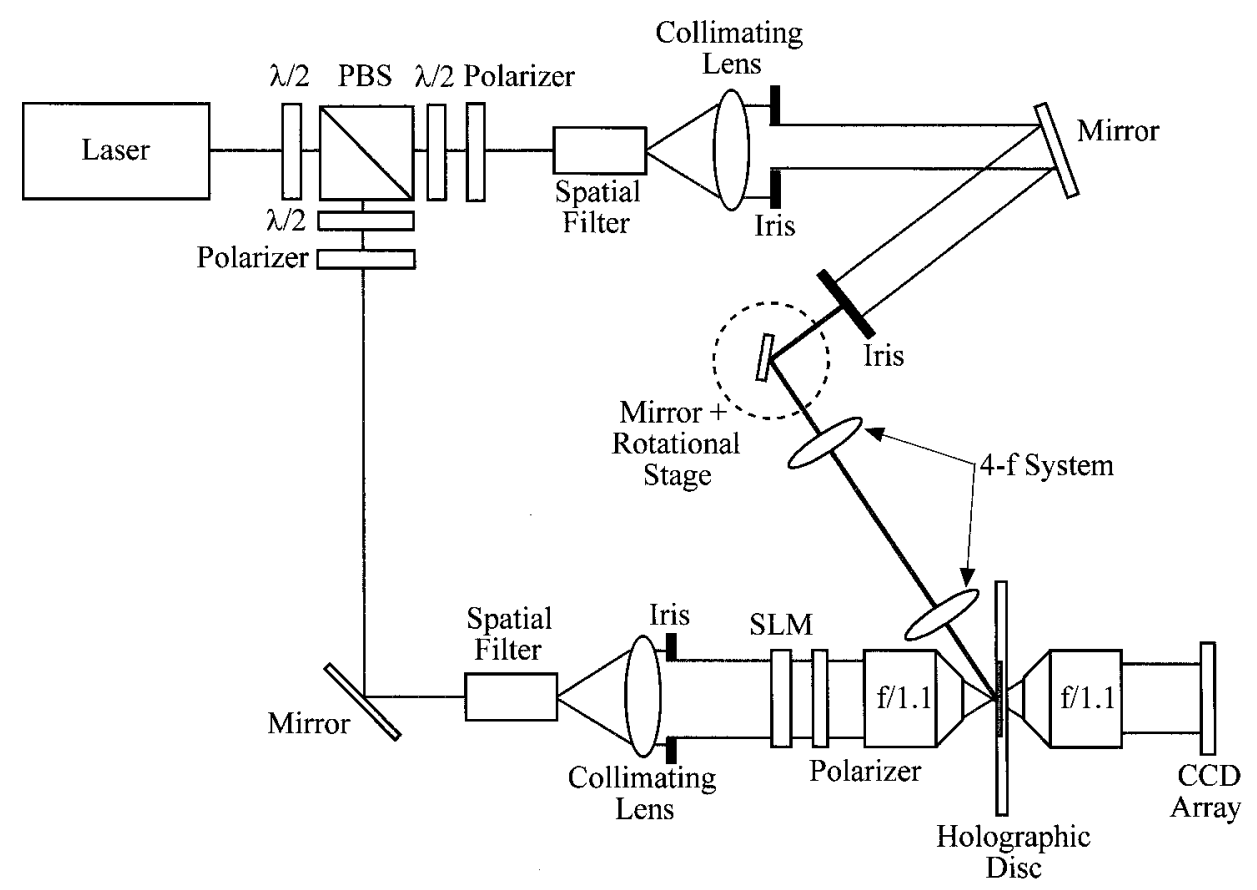

Fig. 2. Schematic diagram of the recording module of holographic ROM.

reference beam through all reference angles. At each reference angle a light exposure is given to the medium. Besides angular multiplexing, we can also rotate the disc to implement peristrophic multiplexing. After all multiplexed holograms are recorded, the disc moves to the next spot and the process starts again until the entire disc is recorded. We use photopolymer as the storage medium (Rhee et al. 1995; Zhou et al. 1996). The disc we record is typically $12 \mathrm{~cm}$ in diameter (standard DVD platter size). The disc is fabricated by laminating a layer of $100 \mu \mathrm{m}$ thick photopolymer onto a glass substrate.

The area recording density of the holographic disc system can be written as

$$
\text { Density }=\left(N_{\mathrm{P}} / A\right) * M \quad\left(\text { bits } / \mu \mathrm{m}^{2}\right)
$$

where $M$ is the number of multiplexed holograms per location, $N_{\mathrm{P}}$ is the number of pixels per hologram, and $A$ is the area of the recording spot. The quantity $N_{\mathrm{P}} / A$ in the above equation is called the Density per Hologram here for obvious reason. The number of multiplexed pages $M$ is determined by the medium thickness and the scanning range of the reference beam (Kogelnik 1969; Amodei and Stebler 1971). To reach a given storage density, it is a good idea to maximize the Density per Hologram, therefore minimizing the required hologram number $M$. Since the diffraction efficiency is proportional 
to $M^{-2}$, smaller $M$ can significantly enhance diffraction efficiency and consequently the data readout rate can be higher. For Fourier plane holograms, the recording area $A$ is determined by the pixel pitch of the SLM. Specifically

$$
A=(2 \lambda F / \delta)^{2}
$$

where $\lambda$ is recording wavelength, $F$ is focal length, and $\delta$ is the pixel pitch of the SLM. The quantity $2 \pi F / \delta$ is the size of the Airy disk of a square pixel having dimension $\delta$. The above expression is valid for a thin disk-like medium, for thick medium there is a correction term associated with thickness (Li and Psaltis 1994). Given a lens with focal length $F$ and diameter $D$, the number of pixels that can be imaged by the lens is

$$
N_{\mathrm{P}}=(\pi / 4) *\left(D / \delta-2 \lambda F / \delta^{2}\right)^{2}
$$

From the above equations it is clear that

$$
\text { Density per Hologram }=\frac{\pi}{4}\left(\frac{D}{2 \lambda F}-\frac{1}{\delta}\right)^{2}
$$

From the Equation (4) it is clear that the density per hologram has only a weak dependence on the pixel pitch $\delta$ of the SLM. The dominating factor is the $f / \#$ of the lens, which is the quantity $F / D$. The density per hologram is almost inversely proportional to the square of the $f / \#$ of the lens. The effect of SLM pixel pitch is important only when $2 \lambda *(f / \#)$ becomes comparable to $\delta$. For example, if $\lambda=532 \mathrm{~nm}, f / \#=1.1$, then the SLM pitch does not play a significant role as long as $\delta$ remains much larger than $1 \mu \mathrm{m}$. To maximize density per hologram, we need a lens with small $f / \#$.

We have designed and fabricated our own imaging optics for use in the disc system. A picture of the imaging optics is shown in Fig. 3. It is about $30 \mathrm{~mm}$ in diameter, with a clear aperture of $11.6 \mathrm{~mm}$. The design spotsize of the lens is about $24 \mu \mathrm{m}$, since this is our SLM pixel size. The distortion of the lens is $0.05 \%$, which is sufficiently good to allow one-to-one match of all the SLM pixels in the field-of-view (FOV) with the detector cells. The performance of the imaging system is shown in Fig. 4, where a page of random data bits is imaged through a glass substrate and captured by a CCD detector. The entire FOV contains 180,000 pixels (480 pixels in diameter) that are one-to-one matched to the detector. The signal-to-noise ratio (SNR) of the transmitted image varies from 10.5 in the center to 7.5 at the edge. These SNR figures correspond to raw bit error rates below $10^{-8}$.

The $f / \#$ of the lens is 1.1 , which gives a recording spotsize of about $0.6 \mathrm{~mm}$, and a Density per Hologram of about $0.5 \mathrm{bits} / \mu \mathrm{m}^{2}$. In other words, when we store one hologram per location without any multiplexing, we 


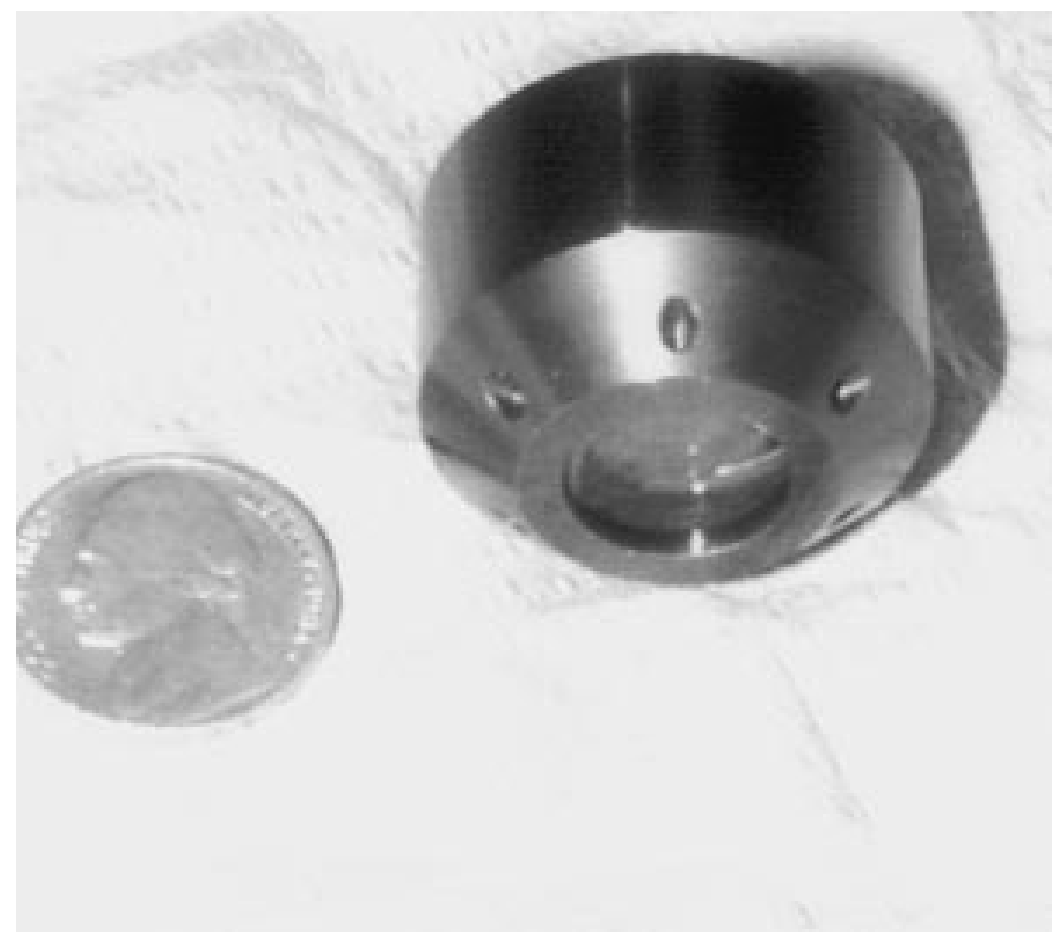

Fig. 3. Picture of the imaging lens designed for HROM.

achieve the surface density of CD-ROM. By multiplexing in a 3D medium, the surface density of the holographic disc can become well above, for example, the DVD density.

Using the components discussed above, we demonstrated the storage of $40 \mathrm{bits} / \mu \mathrm{m}^{2}$ in a $100 \mu \mathrm{m}$ thick photopolymer disc by recording 84 multiplexed holograms over the same location. Figure 5 shows the bit-error-rate (BER) of reconstructed hologram as a function of storage density. At $0.5 \mathrm{bits} / \mu \mathrm{m}^{2}$ density the BER is $10^{-7}$, and at 40 bits $/ \mu \mathrm{m}^{2}$ the BER drops to $10^{-3}$. We have performed a detailed SNR analysis for the experiment, and found that most of the drop in SNR is due to the increase in material scattering from defects and from noise gratings. Some improvement in material quality is needed to bring us to the target raw BER of $10^{-4}$. Also, to obtain storage density higher than $40 \mathrm{bits} / \mu \mathrm{m}^{2}$, a thicker medium is needed. The preferred medium thickness is in the range of $500 \mu \mathrm{m}$ to $1 \mathrm{~mm}$ (Steckman et al. 1998).

\section{The readout module}

The data readout module for holographic disc was shown schematically in Fig. 6. The laser beam (from the top) passes through the modulator and 

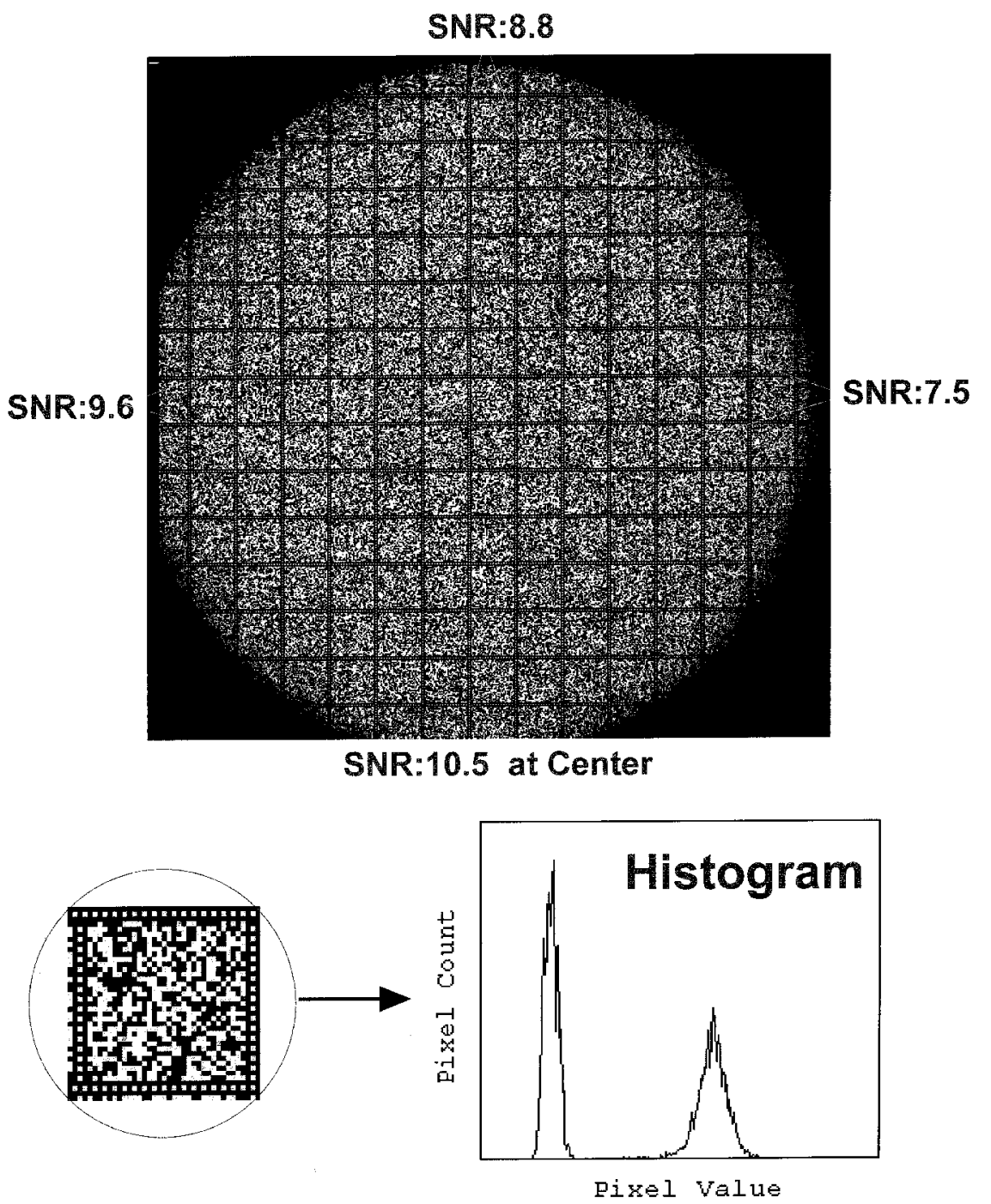

Fig. 4. Performance of the imaging system.

enters the scanner. The two beam deflectors in the scanner can change the laser beam propagation direction along two orthogonal axes in space. The scanner serves two purposes. First, it deflects the laser beam to the appropriate angles in order to address different multiplexed holograms. The entire disc is readout at a particular angle before the deflector changes to the next multiplexing angle. The second role of the scanner is as a compensator in a servo system to correct for image misalignments. This will be explained later in this section. The laser beam is directed by the scanner to the holographic disc which spins continuously at $600 \mathrm{RPM}$. The disc and its spindle motor 


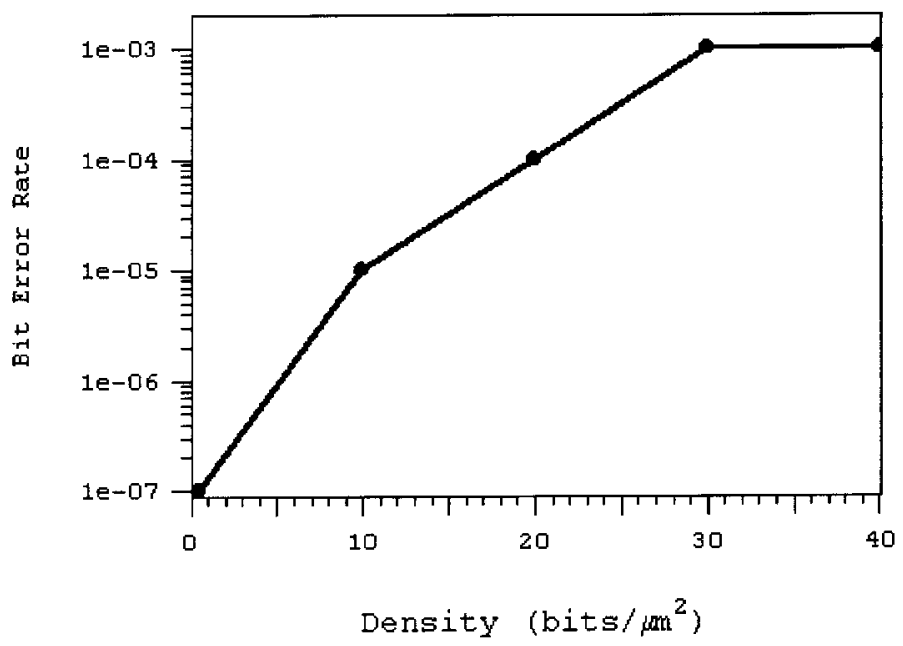

Fig. 5. Measured bit-error-rate as a function of storage density.

can also move linearly to allow the laser pickup to probe different tracks on the disc. A stationary image is read out from the moving disc by pulsing the laser at the appropriate time. A detector array integrates the readout image and converts the photons into electrical signals.

Figure 7 is a picture of the implemented holographic disc reader. We use a diode-pumped green laser as the light source. An external electro-optic modulator is used to pulse the laser. The beam deflectors in the module are

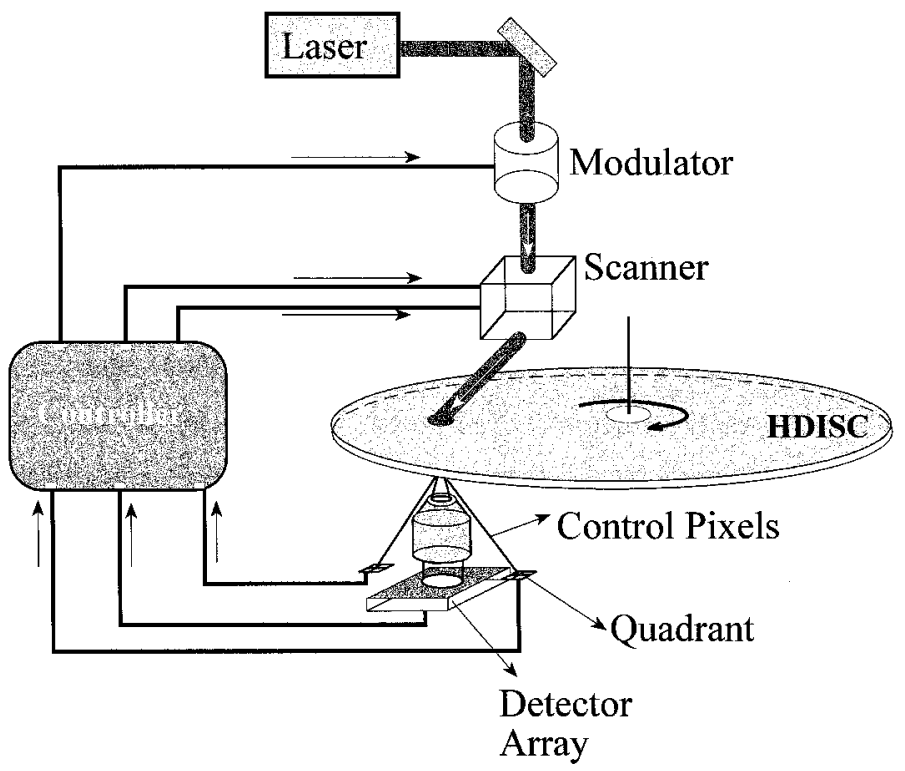

Fig. 6. Schematic of the readout module for holographic disc. 


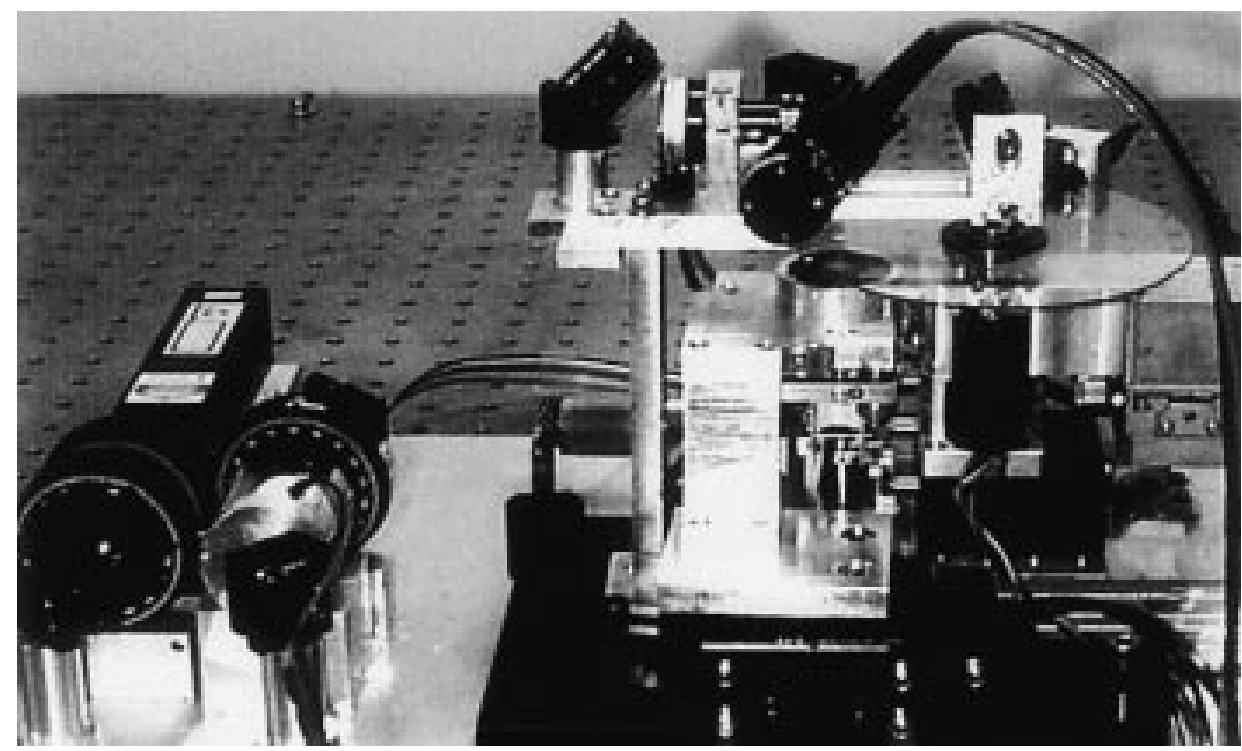

Fig. 7. Picture of the holographic disc reader module.

miniature galvo mirrors. The imaging lens is identical to the ones used in the recording setup. In the following we will first discuss the data readout rate, then we describe the implementation of a servo system to maintain image alignment and good data registration (Zhou et al. 1999).

The readout data rate from the disc can be written as

$$
R=N_{\mathrm{P}} / \tau
$$

Where $N_{\mathrm{P}}$ again is the number of pixels per page, and $\tau$ is the transit time it takes to reach the next hologram. Obviously, the faster the disc spins, the shorter the transit time, and the higher the data rate. At the spinning speed (600 RPM) that we use, the transit time is about $0.17 \mathrm{msec}$. Since $N_{\mathrm{P}}$ is 180,000 in our system, the data readout rate is about $1 \mathrm{Gbit} / \mathrm{sec}$ from the holographic disc. A natural question to ask is why not spin the disc faster, so the transit time is smaller and the optical data rate is even higher? Indeed 600 RPM is not even close to the kind of speed used in hard disc drives, or in high speed CD-ROM drives, which typically can be several thousand RPM. The limit to achieving even greater readout rate is the decrease in dwell time as disc spin rate increases. The time window in which the probe laser can fire and readout a satisfactory image becomes narrower as the disc spins faster.

The dwell time is given by the expression below:

$$
\tau_{\text {DWELL }}=\frac{\Delta x}{2 F \cdot \omega \cdot \sin ((\theta+\phi) / 2) \cos ((\theta-\phi) / 2)}
$$


where $\Delta x$ is the amount of image shift that can be tolerated, $F$ is the focal length of the imaging optics, and $\omega$ is the angular velocity of the disc. $\theta$ and $\phi$ are the angles of incidence of reference and signal beams, respectively. For our system, $\Delta x=2 \mu \mathrm{m}, F=13 \mathrm{~mm}, \omega=62.8 \mathrm{rad} / \mathrm{sec}$, and the dwell time $\tau_{\text {DWELL }}$ is about $2 \mu \mathrm{sec}$.

The number of photons integrated by a detector cell is given by

$$
N_{\mathrm{PHOTONS}}=\frac{P_{\mathrm{LASER}} \cdot \eta \cdot \tau_{\mathrm{DWELL}}}{N_{\mathrm{P}} \cdot 4 \times 10^{-19} \mathrm{~J} / \text { Photon }}
$$

Where $P_{\text {LASER }}$ is the laser power, $\eta$ is the diffraction efficiency, and $N_{\mathrm{P}}$ is again the number of pixels per hologram. For $P_{\text {LASER }}=100 \mathrm{~mW}, \eta=10^{-3}$, the number of integrated photons is $N_{\text {PHOTONS }}=3000$. For a quantum efficiency of 0.3 , the number of signal electrons generated at each detector cell is 900 electrons. The CCD detector and associated amplifiers in our system has a combined noise figure measured at about 250 electrons. Therefore with $2 \mu \mathrm{s}$ dwell time, signal to noise ratio of the detector is about 3.6. Shorter dwell time would make the detector noise contribution more and more significant.

The present $1 \mathrm{~Gb} / \mathrm{sec}$ optical data rate is already orders of magnitude faster than CD or DVD specifications. The high data rate in holographic disc is due to the parallelism in data readout, i.e., data are readout one page (thousands of pixels) at a time. We note that the data rate discussed here is the optical, not electronic, data rate. The present CCD detector in the system has a $16 \mathrm{Mb} / \mathrm{sec}$ output bandwidth. To bring electrical signals out of the holographic disc system at $1 \mathrm{~Gb} / \mathrm{sec}$ requires the use of either a multi-channel CCD, or a high speed CMOS sensor.

If an application calls for data rate greater than $1 \mathrm{~Gb} / \mathrm{sec}$, the solution is to use Q-switched lasers. A Q-switched laser with low average power can have a very high peak power and sufficient pulse energy to boost the data rate by orders of magnitude. As an example, the present system uses a $100 \mathrm{~mW} \mathrm{CW}$ laser pulsed for $2 \mu \mathrm{s}$, corresponding to a pulse energy of $200 \mathrm{~nJ}$. If a pulse laser with $2 \mu \mathrm{J}$ average pulse energy is used as the readout laser, we could spin the disc 10 times faster, work with a dwell time of 200 nanoseconds (which is long compared to the typical pulse width of pulse lasers) and generate sufficient number of signal electrons for good detector SNR.

A different design issue one must address for the readout module is data registration. When the holographic image is readout from the spinning disc and captured by the detector array, the image could be shifted or rotated with respect to the detector cells. The one-to-one match of thousands of pixels with the detector array is not a trivial task, especially when the medium is undergoing rapid mechanical motion. Misregistration of image pixels by the detector array can be caused by disc wobble and by the mechanical deformation in the disc substrate, for example. When these things happen, the reconstructed 

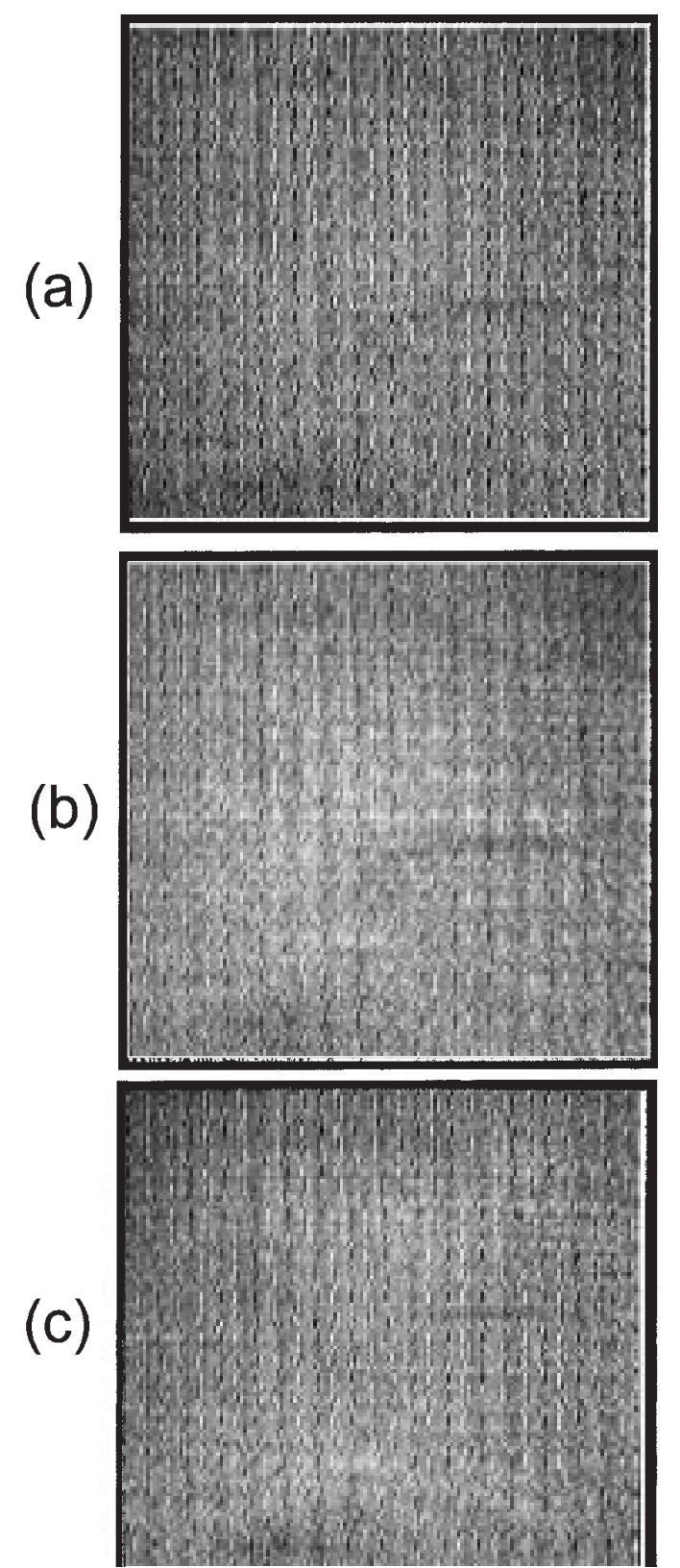

Fig. 8. Raw images read out from the holographic disc. (a) with servo activated; (b) with servo de-activated, showing the effect of image shift; (c) with servo de-activated, showing the effect of image rotation.

holographic image is misaligned with respect to the detector and the bit error rate becomes high. In order to maintain image alignment and good pixel registration, a servo system can be incorporated that detects the readout error in the hologram and actively compensates for the alignment error in real time. 
The detection of alignment error is facilitated by the so-called control pixels and a pair of quadrant detectors in the system. A pair of control pixels was recorded alongside the main image for each hologram. These two extra pixels are directed toward two separate quadrant detectors as illustrated in Fig. 6. When the probe laser is pulsed to read out the hologram, the signal from each quadrant detector is sampled by a controller. The hologram alignment errors are derived from the quadrant signals. The image shift errors (along two orthogonal directions) can be derived by the summation and difference operation of the signals from the four individual cells of a quadrant detector. The image rotational error is derived by comparing the signals from the two different quadrant detectors. Identical signals means no image rotation, a disparity indicates image rotation.

Once the alignment errors in the readout image are detected, they are compensated by the beam deflectors and by adjusting the laser pulse timing. Any rotational error in the detected image is corrected by either advancing or delaying the laser pulse timing. The shift errors are corrected by driving the two independent beam deflectors in a way that forces the image into alignment.

We have implemented a servo mechanism in the holographic disc system. Figure 8 shows three pictures of the same hologram read out from the spinning disc. Figure 8(a) is obtained without the servo mechanism activated; it shows good pixel match and data registration. Figure 8(b) is obtained with the servo de-activated, and the disc tilted slightly. The readout image shows a uniform smear and poor contrast. Figure 8(c) shows the effect of timing error on the readout image. The captured image is slightly rotated relative to the detector array, and the incorrect registration by the detector causes several stripes of low contrast fringes in the image. Figure 9 shows the amount of jitter in the readout image from the holographic disc. Here the intensity profile of small part of a readout image is plotted. In this test, the same holographic image is readout once very $10 \mathrm{~s}$ for 20 times, and the intensity profile of each readout image is plotted on the same graph. It can be seen that the profile stays well-defined, with very small amount of jitter. The result demonstrates the good stability of the holographic disc system. Figure 10 shows an example of the digital image readout from the holographic disc. In this experiment the stored digital data from 20 different holograms are readout and in a sequence and decoded to generate this picture. The digital image was reconstructed faithfully with an error rate of about $10^{-4}$.

\section{Conclusions}

We have reported the implementation of a holographic disc system for readonly memory applications. We discussed the recording module for the 


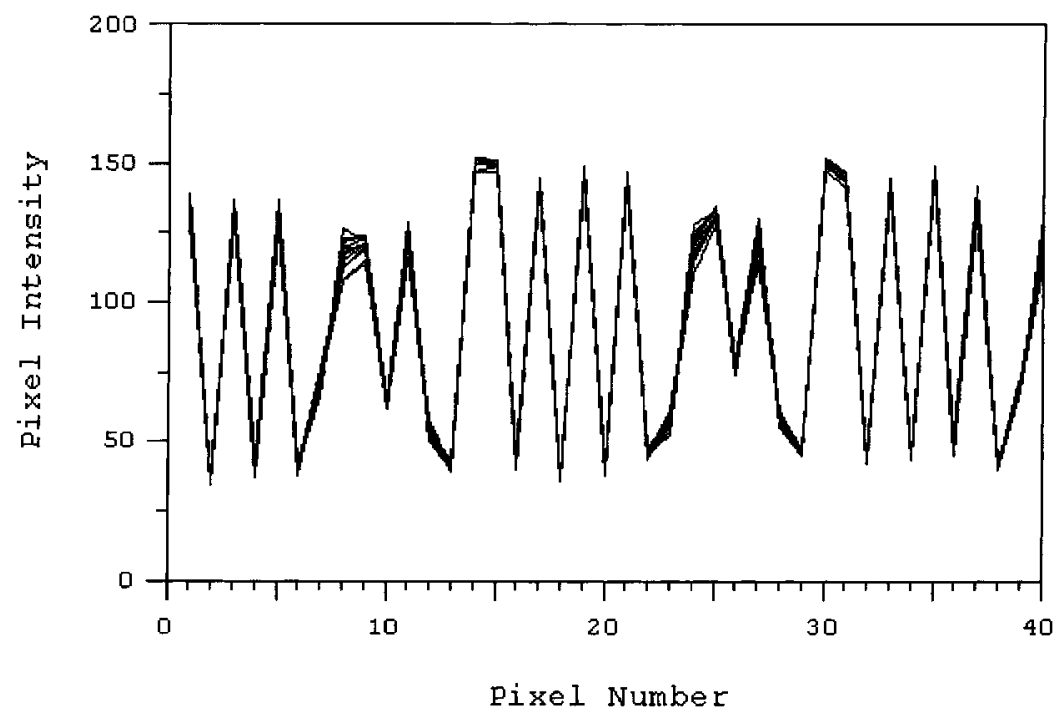

Fig. 9. Measurement of jitter in readout image from the spinning disc.

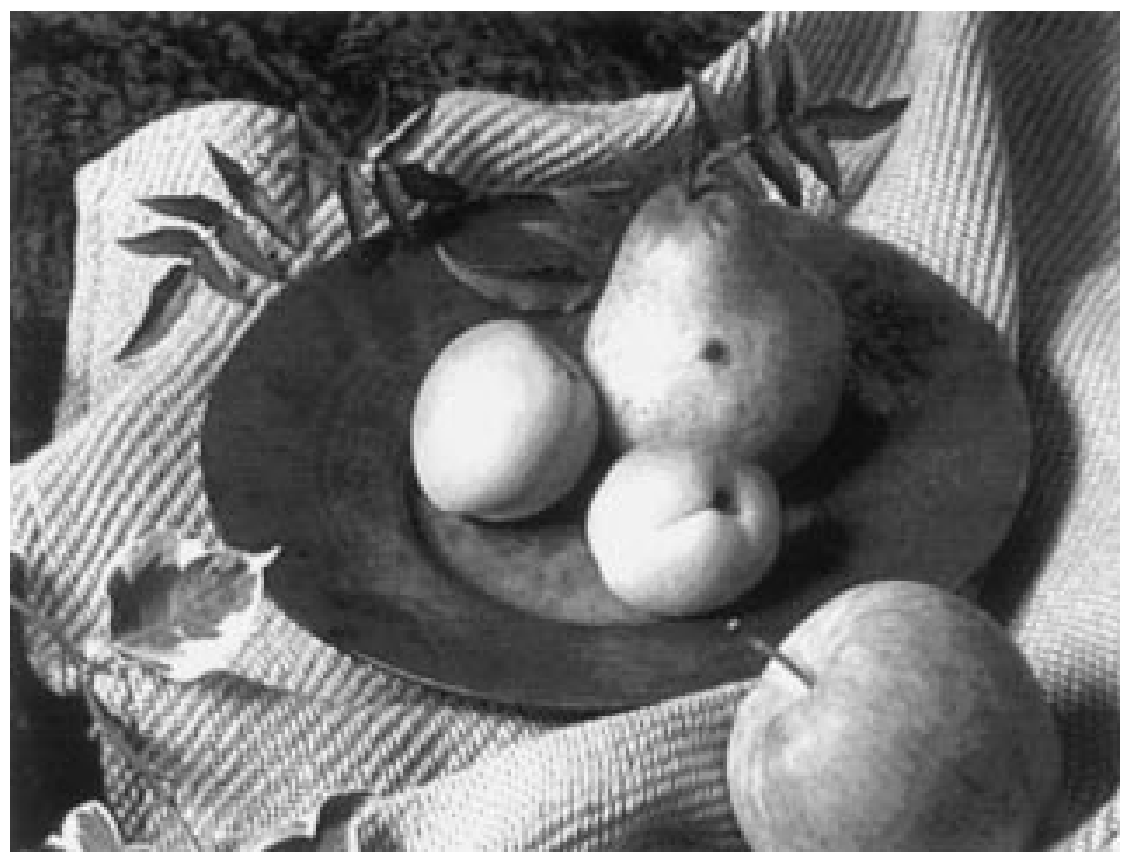

Fig. 10. Example of a digital image readout from the holographic disc.

HROM system and the importance of lens design to achieving good storage density. We have built a compact prototype reader and demonstrated digital data readout from a holographic disc spinning at 600 RPM. 


\section{Acknowledgements}

We thank Allen $\mathrm{Pu}$ and Olga Ivanova for technical assistance during the course of this work. We thank Tillman Stuhlinger for the optical design work, and Gaston Arraya for assistance in the mechanical design of the readout module.

\section{References}

Amodei, J.J. and D.L. Stebler. Appl. Phys. Lett. 18 540, 1971.

Kogelnik, H. Bell Syst. Tech. J. 48 2909, 1969.

Li, H.-Y.S. and D. Psaltis. Appl. Opt. 33(17) 3764, 1994.

Psaltis, D. and F. Mok. Sci. Am. 273(5) 70, Nov. 1995.

Psaltis, D. and A. Pu. Optoelectronics 10(3) 333, 1995.

Rhee, U.-S., H.J. Caulfield, C. Vikram and J. Shamir. Appl. Opt. 34(5) 846, 1995.

Steckman, G.J., I. Solomatine, G. Zhou and D. Psaltis. Opt. Lett. 23(16) 1310, 1998.

Zhou, G., A. Pu, O. Ivanova and F. Mok. (invited paper) "Output interface for holographic memory". In Optoelectronic Interconnects, eds, J. Bristow and S. Tang, Proceedings of SPIE, Vol. 3632, 292-296, 1999.

Zhou, G., A. Pu, O. Ivanova, F. Mok and D. Psaltis. In Proc. Int'l Symp. Opt. Mem., 14. Tsukuba, Japan. 1998.

Zhou, G., Y. Qiao, F. Mok and D. Psaltis. Opt. Photon. News 43, March 1996. 